\title{
ASF1 is required to load histones on the HIRA complex in preparation of paternal chromatin assembly at fertilization
}

\author{
Béatrice Horard", Laure Sapey-Triomphe, Emilie Bonnefoy and Benjamin Loppin * (1)
}

\begin{abstract}
Background: Anti-Silencing Factor 1 (ASF1) is a conserved H3-H4 histone chaperone involved in both ReplicationCoupled and Replication-Independent (RI) nucleosome assembly pathways. At DNA replication forks, ASF1 plays an important role in regulating the supply of $\mathrm{H} 3.1 / 2$ and $\mathrm{H} 4$ to the CAF-1 chromatin assembly complex. ASF1 also provides $\mathrm{H} 3.3-\mathrm{H} 4$ dimers to HIRA and DAXX chaperones for RI nucleosome assembly. The early Drosophila embryo is an attractive system to study chromatin assembly in a developmental context. The formation of a diploid zygote begins with the unique, genome-wide Rl assembly of paternal chromatin following sperm protamine eviction. Then, within the same cytoplasm, syncytial embryonic nuclei undergo a series of rapid, synchronous $S$ and $M$ phases to form the blastoderm embryo. Here, we have investigated the implication of ASF1 in these two distinct assembly processes.

Results: We show that depletion of the maternal pool of ASF1 with a specific shRNA induces a fully penetrant, maternal effect embryo lethal phenotype. Unexpectedly, despite the depletion of ASF1 protein to undetectable levels, we show that asf1 knocked-down (KD) embryos can develop to various stages, thus demonstrating that ASF1 is not absolutely required for the amplification of cleavage nuclei. Remarkably, we found that ASF1 is required for the formation of the male pronucleus, although ASF1 protein does not reside in the decondensing sperm nucleus. In asf1 KD embryos, HIRA localizes to the male nucleus but is only capable of limited and insufficient chromatin assembly. Finally, we show that the conserved HIRA B domain, which is involved in ASF1-HIRA interaction, is dispensable for female fertility.

Conclusions: We conclude that ASF1 is critically required to load $\mathrm{H} 3.3-\mathrm{H} 4$ dimers on the HIRA complex prior to histone deposition on paternal DNA. This separation of tasks could optimize the rapid assembly of paternal chromatin within the gigantic volume of the egg cell. In contrast, ASF1 is surprisingly dispensable for the amplification of cleavage nuclei, although chromatin integrity is likely compromised in KD embryos.
\end{abstract}

Keywords: ASF1, Histone chaperone, Drosophila, Early embryo, Pronucleus, Haploid, Chromatin assembly

\section{Background}

The controlled accessibility of DNA to a variety of proteins is essential for the functioning of eukaryotic genomes. Basic nuclear activities such as gene transcription, DNA repair or DNA replication all require the disassembly of nucleosomes to allow the appropriate

\footnotetext{
*Correspondence: beatrice.horard@univ-lyon1.fr; benjamin. loppin@univ-lyon1.fr Laboratoire de Biométrie et Biologie Evolutive - CNRS - UMR5558, Université Claude Bernard Lyon I, 16, rue R. Dubois - Bât. G. Mendel, 69622 Villeurbanne Cedex, France
}

protein complexes to access specific DNA sequences or regions. However, these dynamic transactions must be compensated by the reassembly of chromatin over nucleosome-depleted regions in order to maintain chromatin integrity. Nucleosome assembly begins with the association of two histone $\mathrm{H} 3-\mathrm{H} 4$ dimers to form a $(\mathrm{H} 3-\mathrm{H} 4)_{2}$ tetramer on DNA. The particle is then completed with the addition of two H2A-H2B dimers to form an octameric nucleosome [1, 2]. From their synthesis in the cytoplasm to their final deposition on DNA, histones are constantly associated with chaperones [3]. Histone 
chaperones form a heterogeneous group of proteins with various functions, such as storage, transport, modification or deposition of histones on DNA $[4,5]$. Originally discovered in the budding yeast, Anti-Silencing Factor 1 (ASF1) is a small, conserved histone chaperone of the $\mathrm{H} 3 / \mathrm{H} 4$ family that is involved in a variety of chromatinrelated functions, such as nucleosome assembly and disassembly, chromatin remodeling, gene silencing or DNA damage checkpoint [6-9]. This diversity of functions reflects the implication of ASF1 in both ReplicationCoupled (RC) and Replication-Independent (RI) histone deposition pathways. Studies in the budding yeast and metazoan have indeed established that ASF1 interacts with subunits of the Cac/CAF-1 (Chromatin Assembly Factor 1; RC) and Hir/HIRA (Histone Regulator-A; RI) complexes, respectively [10-14]. Structural studies have revealed that ASF1 interacts with $\mathrm{H} 3$ and $\mathrm{H} 4$ as dimers by binding the so-called tetramerization interface. This likely regulates the initiation of nucleosome assembly by preventing uncontrolled formation of heterotetramers $[3,9,14-16]$. During DNA replication in animal cells, ASF1 plays a pivotal role in controlling the flux of $\mathrm{H} 3-\mathrm{H} 4$ dimers to the CAF-1 complex, in close cooperation with the replicative helicase subunit MCM2 [17, 18]. Notably, ASF1 knock-down (KD) in Drosophila and human cells blocks the progression of DNA replication forks $[8,17]$. On the other hand, the functional relationship between ASF1 and the HIRA complex has been essentially characterized in the context of transcriptional regulation. For instance, HIRA and ASF1 cooperate for the transcriptional activation of Mef2 target genes in a cell model of mouse muscle differentiation [19]. During the activation of hsp70 (heat-shock protein 70) genes on Drosophila polytene chromosomes, ASF1 presumably cooperates with HIRA and the dATRX/XNP chromatin remodeler to fill nucleosome gaps at these loci [20]. HIRA, UBN1 and ASF1a associate at different regulatory elements over the human genome to allow H3.3 deposition, including active or poised enhancers as well as the transcription start sites of highly transcribed genes [21]. Finally, ASF1 and HIRA cooperate for the transcriptional silencing of heterochromatin regions in fission yeast [22] and the formation of senescence-associated heterochromatin foci in human cells [13].

In Drosophila and mammals, the HIRA complex is critically required for the de novo assembly of nucleosomes on the paternal pronucleus at fertilization [23-25]. Paternal chromatin assembly is a genome-wide, RI assembly process, which begins concomitantly with the eviction of sperm nuclear basic proteins (SNBPs) [26]. We previously reported that both subunits of the Drosophila HIRA core complex (HIRA and YEM, the fly ortholog of mammalian UBN1) specifically localize in the decondensing sperm nucleus where they allow deposition of $\mathrm{H} 3.3-\mathrm{H} 4$ histone dimers on paternal DNA [23, 27, 28]. (H3.3-H4) tetramers must be subsequently complemented with either $\mathrm{H} 2 \mathrm{~A}-\mathrm{H} 2 \mathrm{~B}$ or H2A.Z-H2B histone dimers to finalize the nucleosome assembly process [29]. In this context, we previously reported the unexpected observation that, in contrast to HIRA and YEM, ASF1 was apparently absent from the male pronucleus during nuclear decondensation, thus questioning its role, if any, in this RI assembly process [27]. In addition, during the syncytial phase of embryonic development, ASF1 is known to accumulate in nuclei at each $\mathrm{S}$ phase, but its actual requirement for the rapid amplification of embryonic nuclei has not been investigated. In this study, we have functionally addressed the respective contributions of ASF1 in these two distinct nucleosome assembly pathways during early embryo development.

\section{Results}

\section{Maternal ASF1 is essential for embryo development}

In contrast to most eukaryotes where two asf1 paralogs (asfla and asf1b) are commonly found, Drosophila melanogaster has a single asf1 gene. Hypomorphic asf1 alleles are zygotic lethal at the embryonic or larval stages [30]. To address the function of maternal ASF1, we chose to specifically knock-down asf1 expression in adult female germ cells using transgenic small hairpin RNAs (shRNAs) whose expression can be induced with germline-specific GAL4 drivers [31]. The first tested shRNA (HMC03937), which is expressed from a pValium 20 vector with a minimal $h s p 70$ promoter [31], targets a sequence of 21 nucleotides within the coding region of asf1 (Fig. 1a). We observed that induction of HMC03937 with the ubiquitous Act5C-Gal4 driver was zygotic lethal (all $\mathrm{F} 1$ adults had the $\mathrm{CyO}$ balancer chromosome from the Act5C-Gal4/CyO parent; $n=613$ ). In addition, its specific induction in wing imaginal disks with the vestigial $(v g)$-Gal4 driver efficiently prevented wing development $(100 \% ; n=224)$ suggesting that asf1 is required for cell proliferation in somatic tissues (Fig. 1b). We then turned to another shRNA (GL00171), which targets the predicted 5'UTR of asf1 (Fig. 1a). This shRNA is expressed from the pValium 22 vector that carries a germline-specific promoter and 3'UTR [31]. Accordingly, GL00171 did not cause any somatic phenotype when combined with either Act5C-Gal4 (viable) or vg-Gal4 (normal wing development). Interestingly, however, although oogenesis appeared mostly unaffected by germline expression of GL00171 (using either "MTD-Gal4" or nos-Gal4 drivers), eggs laid by KD females never hatched (Table 1). To verify that this maternal effect, embryonic lethal phenotype was indeed caused by the silencing of asf 1 , we designed a Gal4 inducible, UASP-asf1::V5 rescue transgene that lacks the 


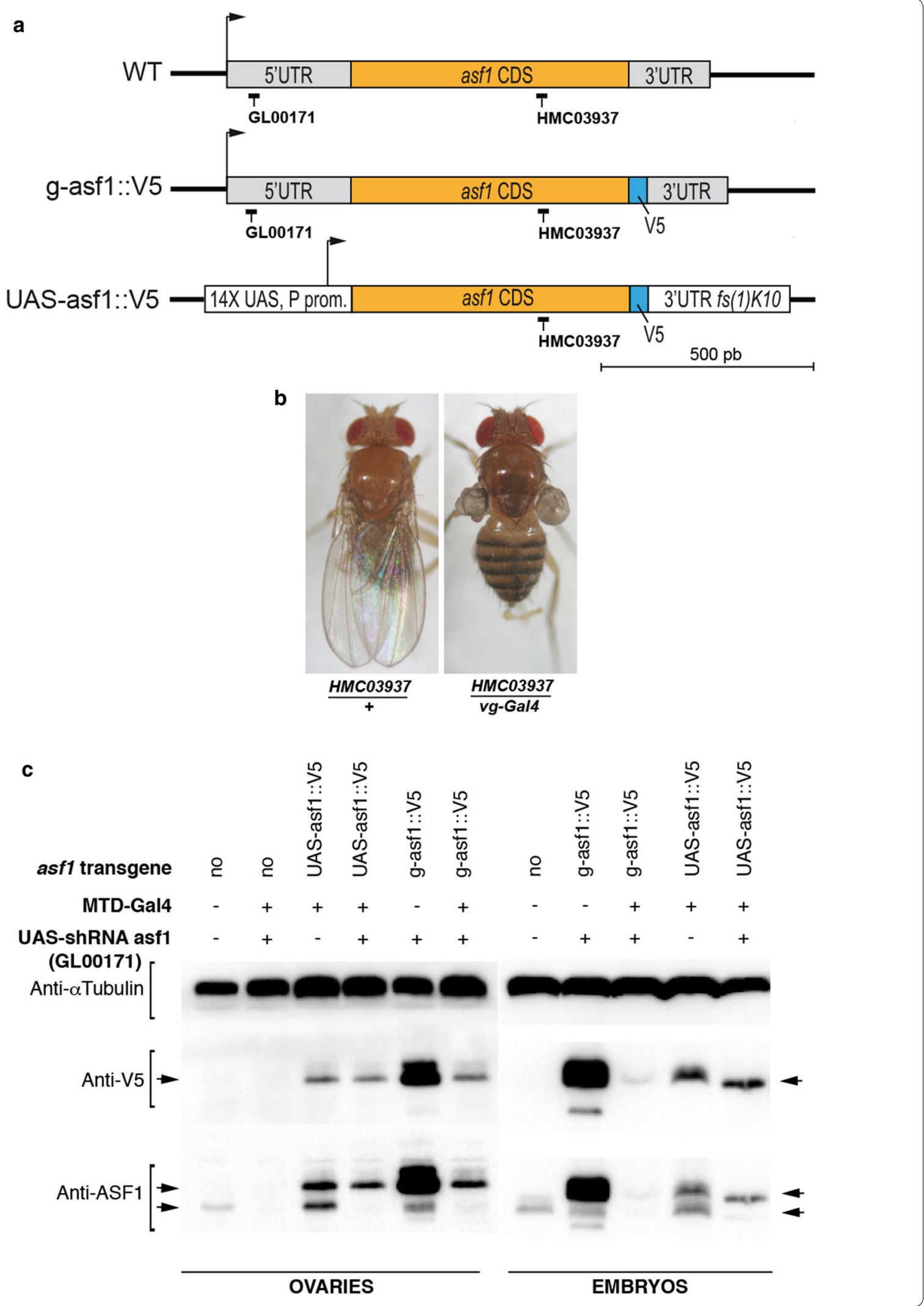


(See figure on previous page.)

Fig. 1 asfl knock-down and rescue experiments. a Schemes of the D. melanogaster asfl gene and rescue transgenes used in this study. The respective target sites of the GL00171 and HMC03937 shRNAs are indicated. b Left: a control P[TRiP.HMC03937]/+ adult showing normal wings. Right: induction of the HMC03937 shRNA in the wing imaginal disk with $v g$-Gal4 results in severe wing atrophy in all tested flies $(n=224)$. c Western blot analysis of endogenous or V5-tagged ASF1 extracted from ovaries or embryos of indicated genotypes. The anti alpha-Tubulin immunostaining was used as a loading control. Note that the UAS-V5-asf1 transgene does not contain the 21-bp target of the asf1 GL00171 shRNA. The anti-ASF1 antibody recognizes both wild-type and tagged versions of ASF1. Arrows indicate specific bands

GL00171 target sequence (Fig. 1a). This transgene fully rescued the fertility of KD females (Table 1). In contrast, a transgene expressing ASF1::V5 under its endogenous upstream regulatory region ( $\mathrm{g}$-asfl::V5; Fig. 1a) poorly rescued the asf1 KD phenotype when females were raised at $25{ }^{\circ} \mathrm{C}$ and had no rescuing effect at $29{ }^{\circ} \mathrm{C}$ (Table 1 ). Western blot analyses confirmed the severe reduction of ASF1 protein levels in KD ovaries or in embryos produced by KD females (hereafter KD embryos) (Fig. 1c). It also revealed that the $g$-asf $1-V 5$ transgene produced massive amounts of ASF1::V5, likely because of a duplication event at the insertion site (not shown). Still, the level of ASF1::V5 produced by this transgene was drastically reduced in KD ovaries and barely detectable in KD embryos, thus confirming the high silencing efficiency of the GL00171 shRNA (Fig. 1c).

Using immunofluorescence, we nevertheless observed the persistence of low levels of ASF1 protein in KD ovaries, thus providing a plausible explanation for the absence of obvious oogenesis defects in KD females. In stage 8-10 egg chambers of control females, ASF1 accumulated in the nucleoplasm of the germinal vesicle (GV), i.e. the oocyte nucleus. This staining was greatly reduced in egg chambers from KD ovaries, but not completely abolished (Fig. 2a). The same distribution was observed using anti-V5 antibodies to detect the tagged version of ASF1. ASF1::V5 was also occasionally detected in individual nurse cell nuclei that were positive for the DNA replication marker PCNA (Fig. 2b) consistent with its implication in RC chromatin assembly [8].

Cytological observations of eggs from KD females revealed frequent chromosome segregation defects during the second meiotic divisions (Fig. 2c, d). Although this indicated a role of ASF1 in the maintenance of oocyte chromosome integrity, the incomplete penetrance of this phenotype implied the existence of additional defects that could account for the fact that asf1 KD embryos never hatched.

\section{Efficient depletion of maternal ASF1 does not abolish nuclear divisions in early embryos}

As previously reported, during the rapid embryonic syncytial divisions, maternal ASF1 accumulated in cleavage nuclei at each $S$ phase but remained in the cytoplasm during mitoses $[27,30]$, in a way similar to DNA replication factors [32, 33] (Fig. 3a). We also confirmed that ASF1::V5 detected with anti-V5 antibodies also enters replicating nuclei at each $S$ phase, consistent with its role in S phase progression (Fig. 3b). Interestingly, ASF1 was never detected in replicating/interphasic nuclei of asf1

Table 1 Fertility tests

\begin{tabular}{|c|c|c|c|c|}
\hline Females & Temperature $\left({ }^{\circ} \mathrm{C}\right)$ & Males & Number of eggs & $\begin{array}{l}\text { Hatching } \\
\text { rate }(\%)\end{array}$ \\
\hline MTD-Gal4 > UAS-shRNA asf1 & 25 & $w^{1118}$ & 1715 & 0 \\
\hline nos-Gal4 > UAS-shRNA asf1 & 25 & $w^{1118}$ & 987 & 0 \\
\hline MTD-Gal4 > UAS-shRNA asf1 & 25 & g-cid-EGFP::cid & 276 & 0 \\
\hline MTD-Gal4 > UAS-shRNA Hira & 25 & g-cid-EGFP:.:cid & 354 & 0 \\
\hline MTD-Gal4 > UAS-shRNA yem & 25 & g-cid-EGFP::cid & 489 & 0 \\
\hline UAS-asf1::V5(62E), UAS-shRNA asf1/TM6 & 25 & $w^{1118}$ & 295 & 91.07 \\
\hline MTD-Gal4 > UAS-asf1::V5(62E), UAS-shRNA asf1 & 25 & $w^{1118}$ & 674 & 92.41 \\
\hline MTD-Gal4 > UAS-asf1::V5(62E), UAS-shRNA asf1 & 29 & $w^{1118}$ & 462 & 80.97 \\
\hline g-asf1::V5(62E), asfi²/asf1 ${ }^{1}$ & 25 & g-asf1::V5(62E), asfi²/asf1 ${ }^{1}$ & 172 & 66.2 \\
\hline$g$-asf1::V5(62E), asf1²/asf1 ${ }^{1}$ & 25 & $w^{1118}$ & 40 & 67.2 \\
\hline g-asf1::V5(62E), UAS-shRNA asf1/TM6B & 25 & $w^{1118}$ & 349 & 78.7 \\
\hline g-asf1::V5(62E), UAS-shRNA asf1 & 25 & $w^{1118}$ & 530 & 74.9 \\
\hline g-asf1::V5(62E), MTD-Gal4 > UAS-shRNA asf1 & 25 & $w^{1118}$ & 1891 & 5.3 \\
\hline g-asf1::V5(62E), MTD-Gal4 > UAS-shRNA asf1 & 29 & $w^{1118}$ & 1314 & 0 \\
\hline
\end{tabular}




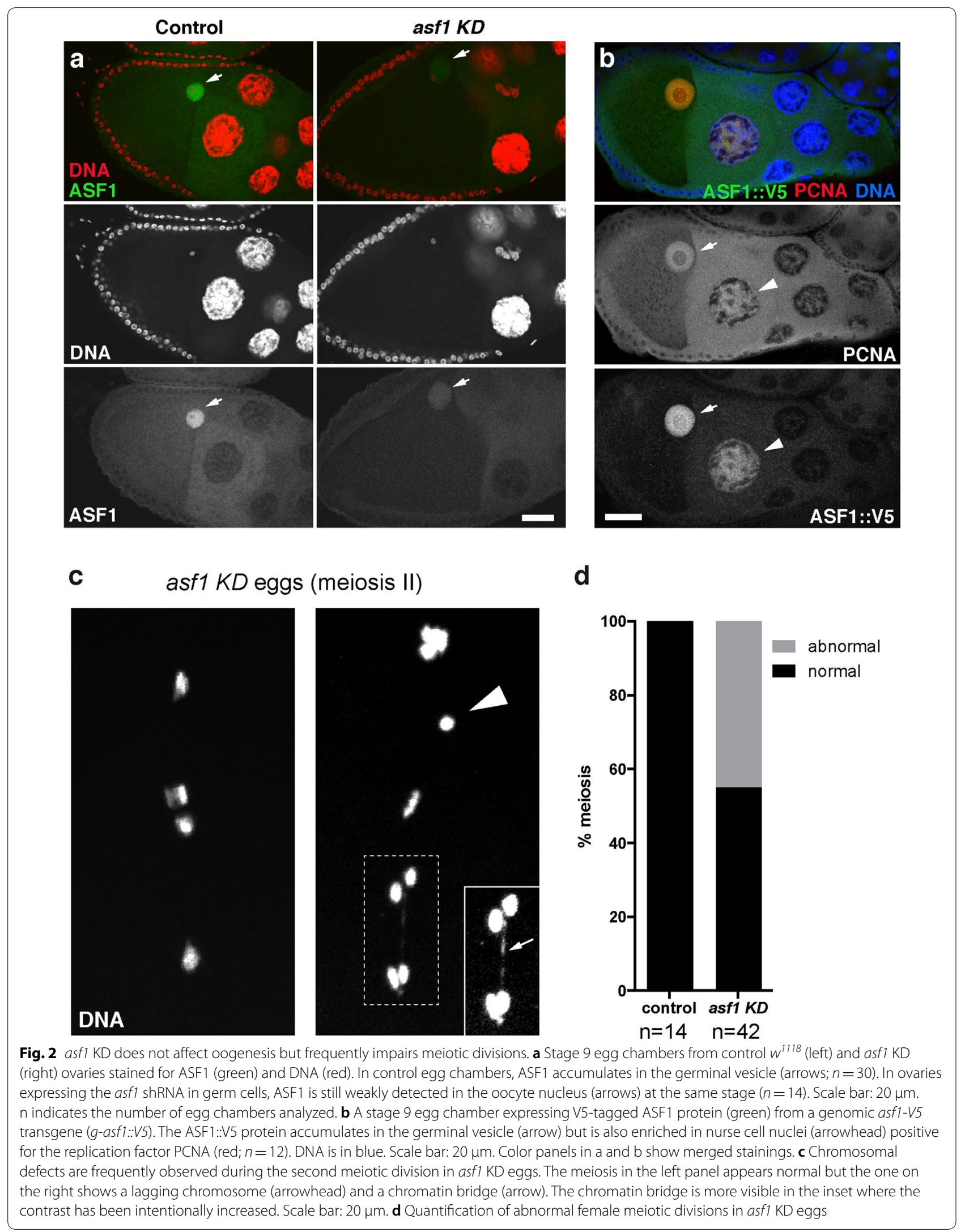



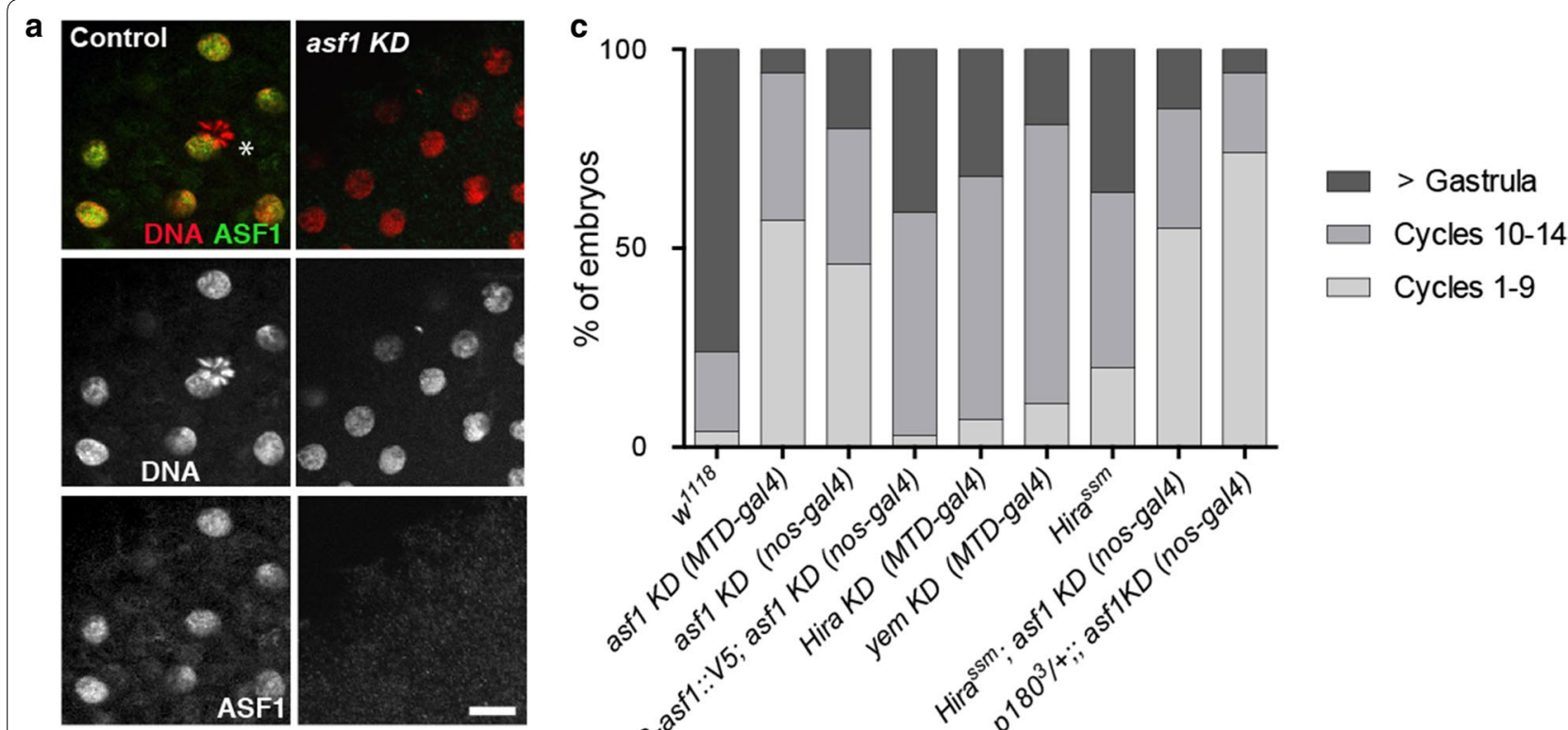

b

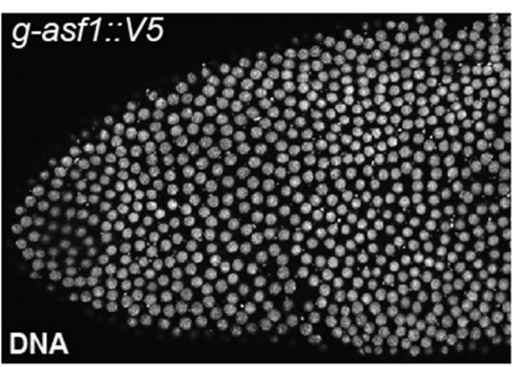

\section{d}

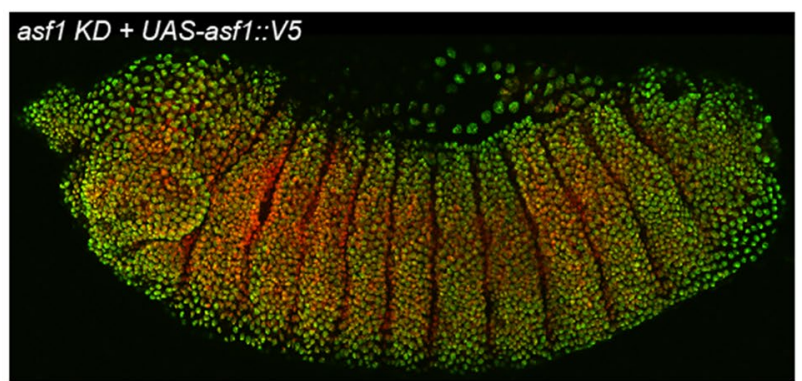

DNA

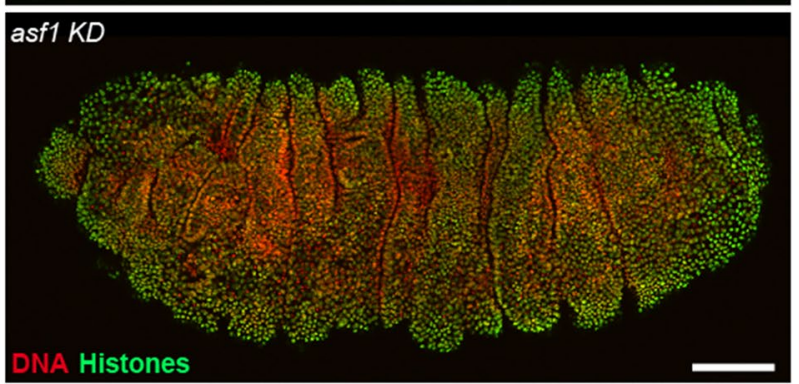

Fig. 3 Depletion of maternal ASF1 does not prevent embryo development. a In control early syncytial embryos (left panels), ASF1 accumulates in nuclei at each synchronous S phase $(n=24)$. Note that ASF1 is not detected in the rosette of condensed chromosomes that form the polar body (asterisk). In embryos from asf1 KD females (right panels), nuclear amplification is observed despite the apparent absence of ASF1 ( $n=28$ ). Scale bar: $10 \mu \mathrm{m}$. b A syncytial blastoderm embryo showing the accumulation of ASF1::V5 in replicating nuclei. ASF1::V5 is expressed from a $g$-asf1::V5 transgene and is detected with an anti-V5 antibody. c Quantification of embryonic phenotypes corresponding to the indicated genotypes. Embryos were collected every $2 \mathrm{~h}$ and then allowed to develop for $2 \mathrm{~h}$ at $25^{\circ} \mathrm{C}$ before fixation and developmental stage determination. A minimum of 150 embryos were scored for each genotype. Corresponding raw data are shown in Additional file 3: Table S1. $\mathbf{d}$ asf1 KD rescue with a UAS-asf1::V5

transgene. Top panel: A representative stage 13 embryo from a asf1 KD female expressing the rescue transgene UAS-asf1::V5 ( $n=62)$. Lower panel: A representative late asfl KD embryo at approximately the same stage showing aberrant segmentation $(n=57)$. Color panels in a and $\mathrm{d}$ show merged stainings. Scale bar: $50 \mu \mathrm{m}$

KD embryos $(100 \%, n=28$; Fig. 3a), indicating that the residual pool of ASF1 present in the germinal vesicle of KD oocytes does not contribute detectable levels of the protein to the egg. The observation of KD embryos that reach the blastoderm stage without detectable level of ASF1 thus demonstrates that this histone chaperone is 
not absolutely required for the amplification of embryonic cleavage nuclei (Additional file 1: Fig. S1). We nevertheless observed that most asf1 KD embryos showed abnormal mitoses, with chromatin bridges frequently connecting dividing nuclei. Developing asf1 KD embryos accumulated aneuploid nuclei that appeared to divide asynchronously (Additional file 2: Fig. S2). These abnormal divisions account for the high proportion of asf1 KD embryos that arrest development before nuclear cycle 9 (Fig. 3c and Additional file 3: Table S1). Finally, the minority of asf1 KD embryos that developed beyond gastrulation all showed highly irregular segmentation compared to viable, UASP-asf1-V5 rescued embryos (Fig. 3d).

\section{ASF1 is required for HIRA-dependent assembly of paternal chromatin}

We made the intriguing observation that developing asf1 KD embryos appeared haploid (Fig. 4a). At cellularization, asf1 KD embryos showed increased nuclear density at the peripheral layer typical of haploid embryos [34] (Fig. 4b). Furthermore, KD embryos did not express a paternally transmitted GFP-Cid transgene, which encodes a fluorescent version of the centromeric histone H3 CID [35], thus indicating the absence of paternal chromosomes (Additional file 4: Fig. S3). We thus examined the fate of paternal chromosomes immediately following fertilization. In Drosophila, sperm DNA is almost entirely packaged with protamine-like, SNBPs [36]. Immediately following the delivery of the sperm nucleus into the egg cytoplasm, SNBPs are replaced with maternally provided histones and the paternal nucleus begins to decondense [37]. By the time of pronuclear apposition in wild-type eggs, both male and female pronuclei are similar in size and almost indistinguishable. In striking contrast, in all asf1 KD eggs observed at the apposition stage $(n=46)$, the male pronucleus appeared abnormally small and condensed (Fig. 4c). This highly specific defect was reminiscent of the maternal effect phenotype associated with mutations in the Hira or yemanuclein (yem) genes, which encode the core subunits of the HIRA complex $[28,38]$. We thus analyzed de novo assembly of paternal chromatin in asf1 KD eggs using anti-acetylated H4 (H4act) antibodies [23]. In wild-type eggs, before the onset of the first $\mathrm{S}$ phase, anti-H4act antibodies brightly and specifically stained the male nucleus. In contrast, acetylated $\mathrm{H} 4$ was only weakly detected in the male nucleus of asf $1 \mathrm{KD}$ eggs, thus confirming the defective paternal chromatin assembly (Fig. 5a). The limited but consistently observed H4act staining in the male nucleus of asf1 KD eggs contrasted with Hira KD or yem KD where paternal chromatin assembly was almost completely abolished (Fig. 5a,d). In addition, paternal chromatin in asf1 KD eggs remained closely associated with maternal chromosomes and was thus more prone to interfere with the first division (Fig. 5b,c) compared to the inert, almost histone-free male nucleus typically observed in the absence of HIRA or YEM $[28,39]$. Along the same line, asf1 KD females produced $56.6 \% \quad(n=258)$ of embryos that never reached the blastoderm stage whereas Hira KD or yem KD females produced only $5.7 \%(n=579)$ and $10.8 \%$ $(n=443)$ early arrested embryos, respectively (Fig. 3c and Additional file 3: Table S1). Nonetheless, the high proportion of early arrest asf $1 \mathrm{KD}$ embryos remained essentially unchanged when asf1 KD females were also homozygous for $\mathrm{Hira}^{\text {ssm }}$, a strong loss of function mutation in the Hira gene [23] (Fig. 3c and Additional file 3: Table S1). This confirms that asf1 KD embryos, beside their pronuclear phenotype and haploid development, have additional defects during the syncytial phase of embryogenesis. Accordingly, the nuclear layer at the surface of asf1 KD blastoderm embryos typically appeared irregular, with multiple patches of nuclear fallout, a phenotype indicative of the presence of damaged nuclei (Fig. 4b). Interestingly, when the asf1 shRNA was induced in females heterozygous for a null allele of the Caf1 $p 180$ gene $\left(+/ p 180^{3}\right.$; GL00171/ nos-Gal4), which encodes the large subunit of the Drosophila CAF-1 complex, the proportion of early arrested embryos increased to $73.6 \%$, compared to $48.3 \%$ for control +/FM7c; GL00171/nos-Gal4 females (Fig. 3c and Additional file 3: Table S1). This genetic interaction between asf1 and Caf1 p180, which was previously reported in another context [40], thus supports a nonessential but significant role of ASF1 during RC chromatin assembly in cleavage nuclei, likely by controlling the supply of $\mathrm{H} 3$ and $\mathrm{H} 4$ to the CAF-1 complex at replication forks.

\section{ASF1 is not directly involved in paternal chromatin assembly}

The implication of ASF1 in paternal chromatin assembly was not anticipated since we previously reported that ASF1 was absent from the decondensing male nucleus undergoing chromatin remodeling [27]. To confirm this result, originally obtained using the anti-ASF1 antibody, we stained eggs from $g$-asf1-V5 transgenic females with an anti-V5 antibody. Although the anti-V5 staining was generally weaker than the anti-ASF1 staining in early embryos, we observed in most cases $(83 \% ; n=12)$ the expected presence of ASF1-V5 in both pronuclei shortly before apposition, at the onset of DNA replication. However, ASF1-V5 was never detected in the decondensing male pronucleus observed during meiosis II $(n=13)$, thus confirming that ASF1 does not reside in the male nucleus 

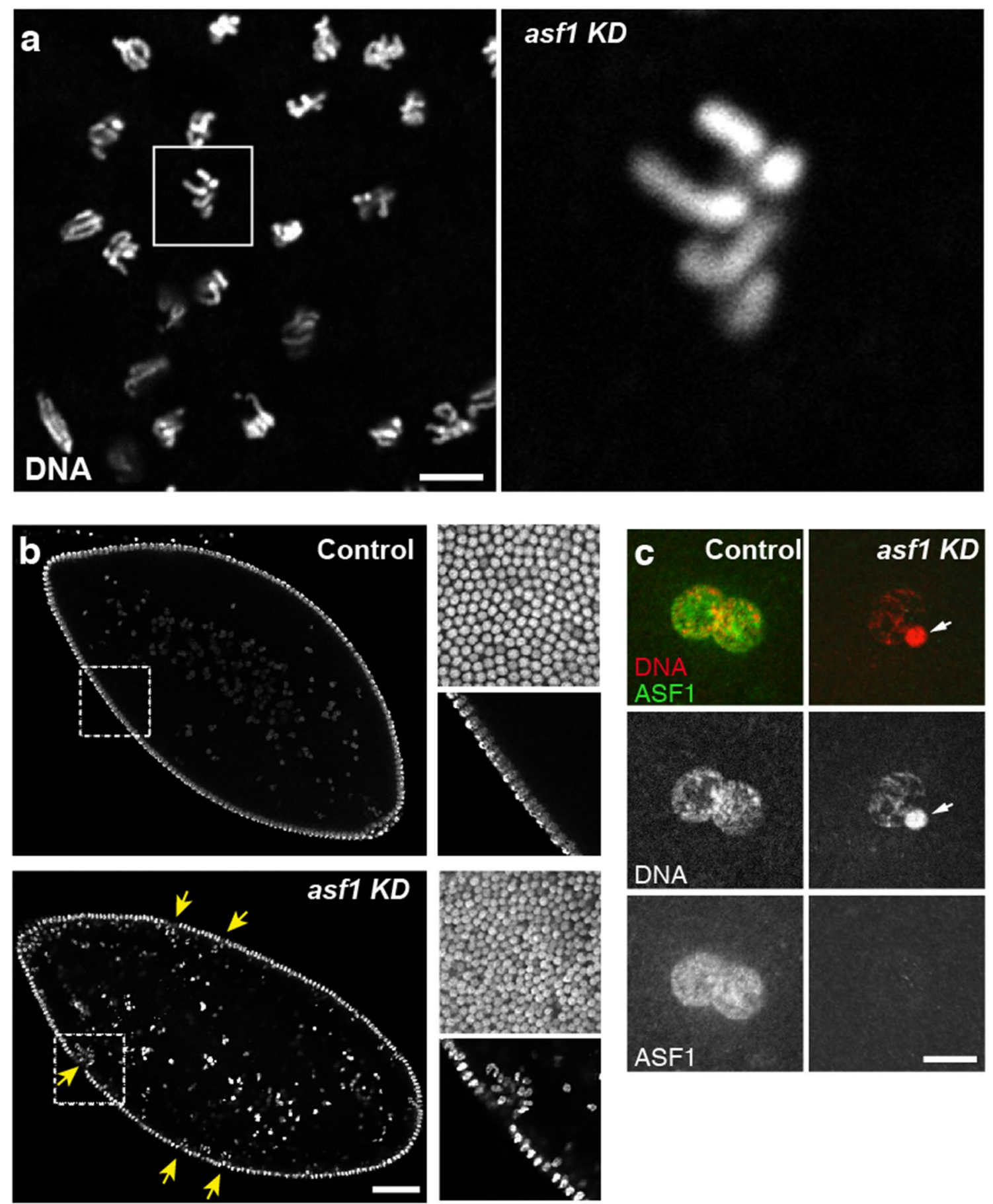

Fig. 4 Gynohaploid development of asf1 KD embryos. a A group of syncytial nuclei in metaphase from an asf1 KD embryo. These nuclei are haploid (four chromosomes), as visible in the close-up. Scale bar: $10 \mu \mathrm{m}$. b Confocal sections of cellularized asf1 KD $(n=67)$ and control blastoderm ( $n=83)$ embryos. Insets were taken at the surface of the same embryo (top) or in the same plane than the main image (bottom). asf1 KD embryos typically show irregular organization of the nuclear layer, with frequent patches of nuclear sinking (yellow arrows). Note the doubled nuclear density at the surface in haploids compared to the diploid control. Scale bar: $50 \mu \mathrm{m}$. c In control eggs (left) at pronuclear apposition, ASF1 (green) is abundant in both apposed pronuclei $(100 \% ; n=20)$. In asf1 KD eggs (right) at the same stage, no signal is detected in apposed pronuclei (0\%; $n=24)$. Note that the female pronucleus appears normal while the male pronucleus fails to decondense (arrowhead). Scale bar: $10 \mu \mathrm{m}$. Color panels in c show merged stainings 


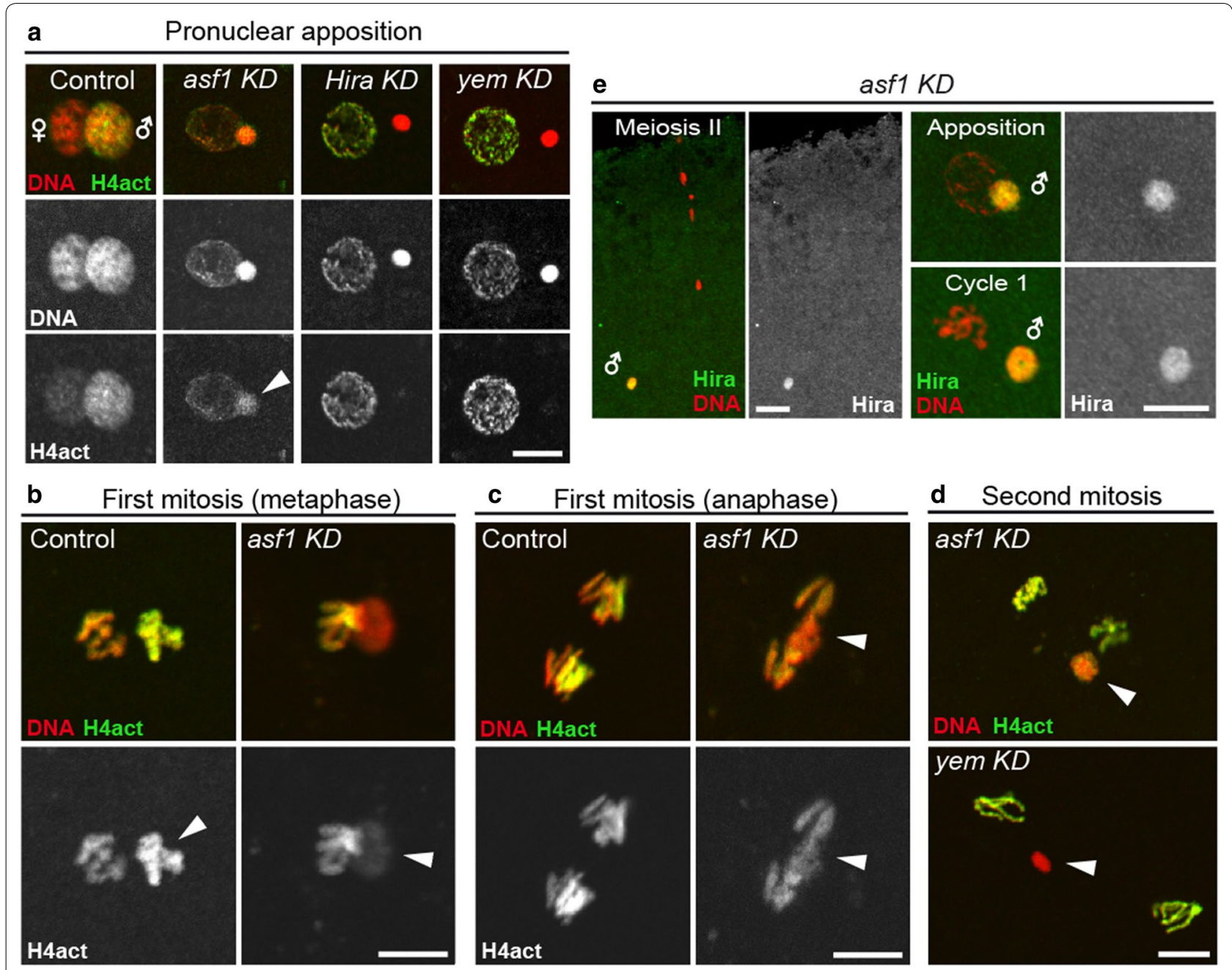

Fig. 5 ASF1 is required for HIRA-dependent assembly of paternal chromatin. a Confocal images of eggs at pronuclear apposition stained for acetylated histone $\mathrm{H} 4$ (H4act) and DNA. Female genotypes are indicated. In asf1 KD eggs, limited de novo histone deposition is observed in the male nucleus (arrowhead). In Hira KD or yem KD eggs, histones are barely detectable in the male nucleus. Scale bar: $10 \mu \mathrm{m}$. b Metaphase of the first zygotic mitosis. In control embryos (left), paternal chromosomes (arrowhead) stain brightly with anti-H4act antibodies and appear greenish in the merged image. In asf1 KD embryos, the male nucleus (arrowhead) fails to condense into mitotic chromosomes and stains weakly for $\mathrm{H} 4 \mathrm{act}$. Scale bar: $10 \mu \mathrm{m}$. c Anaphase of the first zygotic mitosis in control (left) and asf1 KD embryos (right). In the control, paternal chromosomes are still distinguished by the anti-H4act staining and appear yellowish. In asf1 KD embryos, paternal chromatin (arrowhead) lags behind migrating maternal chromosomes. Scale bar: 10 m. d Second mitotic division. In asf1 KD embryos, the male nucleus (arrowhead) typically appears partially decondensed and stains weakly with anti-H4act antibodies (orange color). In contrast, the male nucleus in yem KD embryos remains condensed and free of H4act (it thus appears red). Scale bar: $10 \mu \mathrm{m}$. e HIRA localizes and persists in the male nucleus in the absence of ASF1. Left: Anti-HIRA antibodies normally stain the male nucleus in asf1 KD egg during female meiosis II. Right: HIRA remains abundant in the male nucleus in asf1 KD eggs during pronuclear apposition and the first zygotic mitosis. HIRA was detected in all eggs or cycle 1 embryos analyzed (100\%; $n=15)$. Color panels show merged stainings. Scale bar: $10 \mu \mathrm{m}$

during RI nucleosome assembly (Additional file 5: Fig. S4). We then wondered whether the distribution of HIRA itself was affected in asf1 KD eggs. In wild-type eggs, HIRA specifically accumulates in the decondensing nucleus where it persists until pronuclear apposition $[23,27]$. We observed that, despite the observed defective chromatin assembly, HIRA localized normally in the male nucleus in asf1 KD eggs. Furthermore, HIRA remained strongly enriched in the condensed male nucleus, even after the onset of the first mitosis (Fig. 5e). Our results thus strongly suggest that ASF1 is critically required to hand over histones to the HIRA complex prior to its translocation to the male pronucleus, while ASF1 itself does not reside on chromatin during the assembly process. 
The HIRA B domain is not essential for paternal chromatin assembly

Both structural and functional analyses have collectively established that ASF1 provides $\mathrm{H} 3.3-\mathrm{H} 4$ dimers to HIRA through a direct physical interaction involving a small, evolutionary conserved domain of 37 amino-acids called the HIRA B domain [13, 19, 42-44]. To address the functional contribution of this domain during paternal chromatin assembly, we generated flies expressing a HIRA::Flag protein bearing a small deletion in the $\mathrm{B}$ domain (Hira $[\Delta B]$-Flag) (Fig. 6a,b). The HIRA[ $\Delta B]$ ]:Flag recombinant protein lacks 16 residues (428-443) that correspond to the minimal peptide required for ASF1 binding [43]. Surprisingly, the Hira[ $\triangle B]$-Flag transgene fully rescued the fertility of females homozygous for a null allele of Hira (Fig. 6a). Cytological examination of rescued eggs confirmed that the deleted region is dispensable for paternal chromatin assembly. We noticed, however, that the male nucleus in rescued Hira $[\Delta B]$-Flag eggs observed at pronuclear apposition appeared on average about $30 \%$ smaller in diameter than its female counterpart (Fig. 6c,d), suggesting that the rate of paternal chromatin assembly and nuclear decondensation were suboptimal in this context. To evaluate the impact of the $\triangle B$ deletion on the ability of HIRA to interact with ASF1 in vivo, we performed co-immunoprecipitation experiments using ovarian extracts. Interestingly, the $\Delta B$ deletion did not affect the HIRA-ASF1 interaction to appreciable levels (Fig. 6e). Our results thus suggest that these proteins can rely on additional or different domains for their interaction in the peculiar context of the female germline.

\section{Discussion}

\section{ASF1 contributes to the maintenance of chromatin} integrity during cleavage divisions

In contrast to HIRA and YEM, the two known core HIRA complex proteins in Drosophila, zygotic expression of ASF1 is essential for development and viability. Its well-established role in $S$ phase progression $[8,17$, 45 ] is indeed likely critical in Drosophila proliferating cells, as illustrated in this work by the dramatic impact of asf1 KD on wing development. Unexpectedly, the depletion of ASF1 to undetectable levels in early embryos did not abolish cleavage nuclear divisions. As a matter of comparison, knocking down a catalytic subunit of the DNA polymerase epsilon complex completely blocks development as early as the first zygotic mitosis [46]. Thus, despite the abundance of ASF1 in replicating embryo nuclei, it is not absolutely required for nuclear divisions. We thus speculate that, in the highly peculiar context of the early Drosophila embryo, the absence of ASF1 is at least partially compensated by other histone chaperones. Interestingly, ASF1 appears equally dispensable for RC chromatin assembly in Xenopus egg extracts, where other $\mathrm{H} 3-\mathrm{H} 4$ histone chaperones, such as N1/N2, could possibly provide replicative histones to the CAF-1 complex [47]. In addition, MCM2, a conserved subunit of the minichromosome maintenance helicase, can associate with both parental and newly synthesized histones $\mathrm{H} 3$ and $\mathrm{H} 4$, opening the possibility that it supplies $\mathrm{H} 3-\mathrm{H} 4$ dimers to the CAF-1 complex at replication forks $[17,18,48]$. In human cells, the histone acceptor property of ASF1 has also been proposed to facilitate the unwinding of parental chromatin ahead of the replication fork [17]. In any case, our study suggests that the cooperation of ASF1 with the CAF-1 complex in early Drosophila embryos, although not absolutely essential, is nevertheless important for the maintenance of chromatin integrity during the rapid succession of cleavage divisions.

\section{ASF1 is essential for the decondensation of the male pronucleus}

In this study, we have established that Drosophila ASF1 is critically required for histone deposition in the male pronucleus. Although de novo assembly of paternal chromatin was generally initiated in asf1 KD eggs, the male pronucleus only faintly stained with anti-histone antibodies and nuclear decondensation was severely impaired. We have previously reported that in eggs from yem mutant females that were partially rescued with

(See figure on next page.)

Fig. 6 The HIRA B domain is dispensable for sperm chromatin remodeling. a Representation of HIRA::Flag proteins expressed from transgenes used in c-e. The position of WD40 repeats and B domain are shown. The ability of each corresponding transgene to rescue the fertility of homozygous Hira ${ }^{H R 1}$ females (embryo hatching rate) is indicated. b Alignment of human $(H s)$ or Drosophila $(D m)$ HIRA B domains. Conserved residues are highlighted in red. The deleted residues in HIRA[ $\triangle B]$-Flag are indicated. $\mathbf{c}$ Representative images (merged DNA and H4act stainings) of pronuclear apposition (upper panels) and first zygotic anaphase (lower panels) from the indicated females. Scale bar: $10 \mu \mathrm{m}$. $\mathbf{d}$ Quantification of pronuclear size at apposition for the indicated genotypes are represented as a scatter plot. Horizontal lines represent means. The number of pronuclei analyzed is shown under the graph. In Hira ${ }^{H R T}$, Hira ${ }^{\Delta B}$...Flag flies, the size of male pronucleus relative to the female counterpart is significantly smaller than in control flies (Unpaired t test: $p<0.0001$ Hira ${ }^{\Delta B}$..Flag versus wild type and $p=0.0004$ Hira ${ }^{\Delta B}$. .Flag versus Hira::Flag). e Co-immunoprecipitation experiments performed with anti-FLAG (HIRA) and anti-V5 (ASF1) on ovarian extracts from females carrying the indicated transgenes and mutant alleles. Deletion of the HIRA B domain does not abolish the ASF1-HIRA interaction in ovaries 


\section{a}

B domain

NEANQRPISKQTETRTKDGKRRITPMFIPLHEDGPTS

Hira ${ }^{H R 1}$ rescue

(hatching rates)

HIRA::FLAG

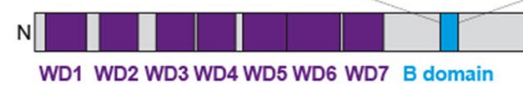

C $89 \%(n=188)$

WD1 WD2 WD3 WD4 WD5 WD6 WD7 B domain

3xFLAG

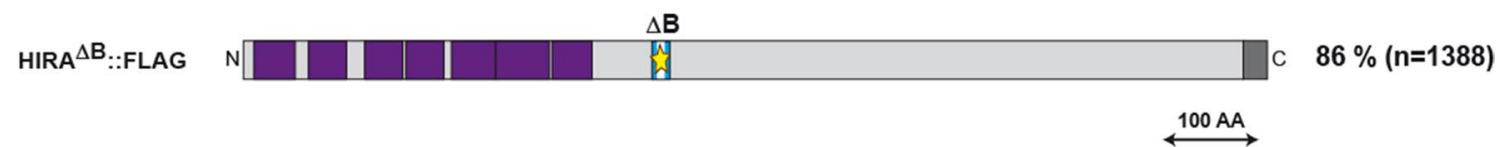

b Hs HIRA (439-475) EDIRKNLLKKOVETRTADGRRRTTPLCIAQLDTGDFS Dm HIRA (414-450) NEANORPISKOTETRTKDGKRRITPMFIPLHEDGPTS Dm HIRA::FLAG NEANQRPISKQTETRTKDGKRRITPMFIPLHEDGPTS Dm HIRA ${ }^{\Delta \mathbf{B}}:$ :FLAG NEANQRPISKOTET..............
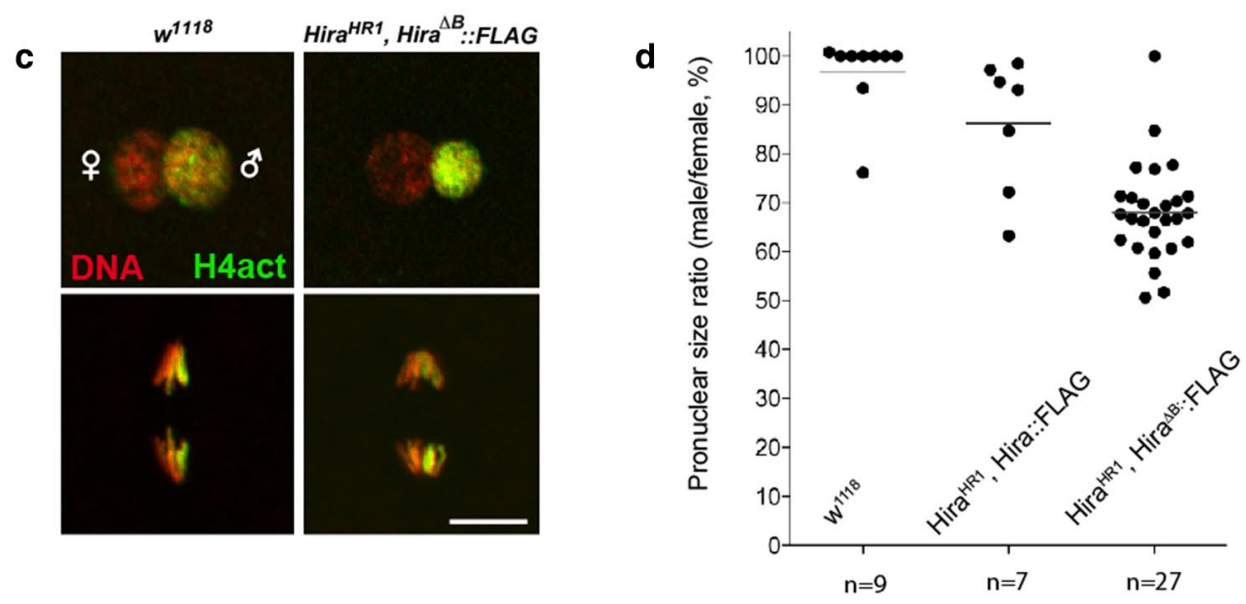

e

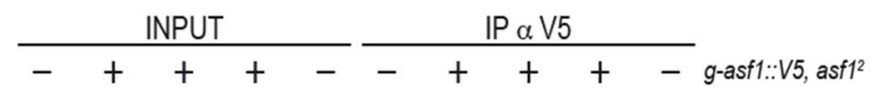

$+-\quad+-\quad+\quad-\quad+\quad-\quad-$ g-Hira::FLAG
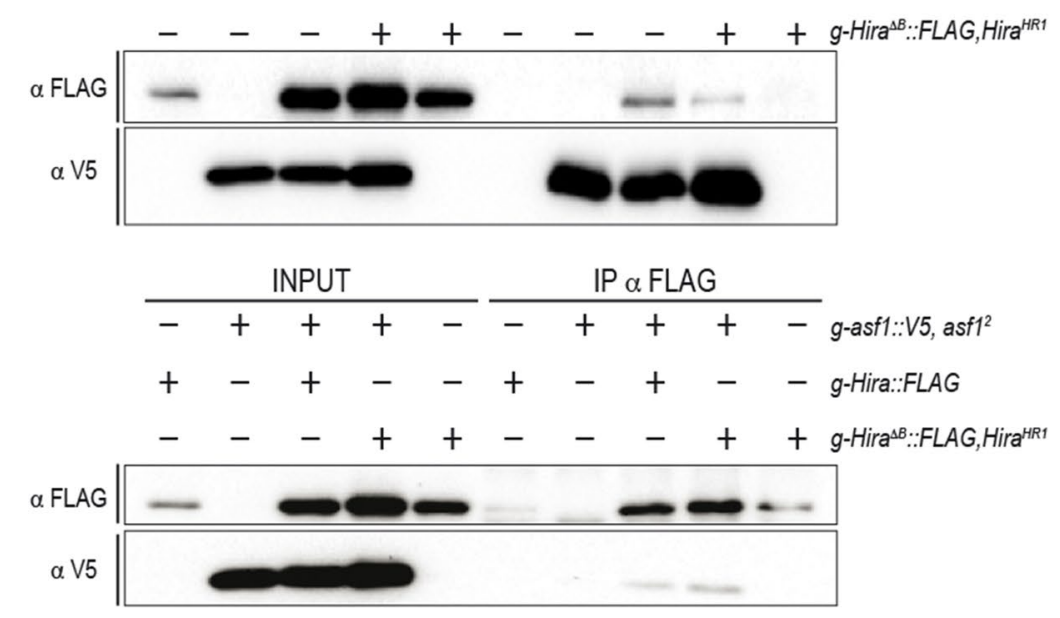
a transgene expressing YEM at suboptimal levels, the male pronucleus incorporated at least some histones but failed to decondense normally [28]. Similarly, in asf1 KD eggs, the HIRA complex is still capable of limited nucleosome assembly in the male pronucleus, but this residual activity is not sufficient to ensure the timely assembly of paternal chromatin and pronuclear decondensation. Interestingly, it has been recently shown that depletion of ASF1 from Xenopus egg extracts prevents de novo nucleosome assembly on prepared mouse sperm nuclei [49]. The results obtained with this artificial system suggest that ASF1 could play a conserved function in assisting the HIRA complex during paternal chromatin assembly.

Importantly, we have also confirmed that ASF1 is not detectable in the male nucleus, despite its presence later on in replicating pronuclei. This paradoxical situation indicates that although ASF1 is required for male pronuclear formation, it does not directly participate in the proper assembly of H3.3-containing nucleosomes on sperm DNA. The apparently normal distribution of HIRA in the decondensing sperm nucleus of asf1 KD eggs also indicates that the phenotype is not caused by the incapacity of the HIRA complex to enter the male pronucleus. We instead favor a model where ASF1 is required to load the HIRA core complex with $\mathrm{H} 3.3-\mathrm{H} 4$ dimers before its translocation to the fertilizing sperm nucleus. In the absence of maternal ASF1, preloading of the HIRA complex with $\mathrm{H} 3.3$ and $\mathrm{H} 4$ is most likely impaired, which prevents efficient assembly of paternal chromatin. This situation is reminiscent of the male pronuclear phenotype observed in eggs from Hira $^{\text {ssm }}$ females, where the HIRA protein with a R225K substitution localizes normally to the male nucleus but fails to assemble chromatin [23]. Similarly, depletion of H3.3 in mouse zygotes impairs paternal chromatin assembly but does not prevent HIRA to localize to the paternal pronucleus [24].

\section{Distinct functions of ASF1 and HIRA at fertilization}

Our demonstration that ASF1 is not directly involved in histone deposition in the male pronucleus also corroborates with previous findings. For instance, in H3.3 depleted salivary glands, although HIRA, XNP and ASF1 are all initially recruited at induced $H s p 70$ genes, only HIRA and XNP persist on chromatin after heatshock, suggesting that ASF1 does not reside on DNA during transcription-coupled nucleosome assembly [20]. In addition, it has been proposed that in human cells, the HIRA core complex (HIRA, UBN1 and Cabin1) fills nucleosome gaps by directly interacting with naked DNA, a property not shared by ASF1a [41]. Finally, HIRA, UBN1 and Cabin1, but not Asf1a and Asf1b, are recruited at sites of DNA damage to restore chromatin after DNA repair [50].
The assembly of paternal chromatin provides yet another dramatic illustration of the different properties of ASF1 and HIRA complex proteins with respect to histone deposition on DNA. Our study strongly indicates that the handover of $\mathrm{H} 3.3-\mathrm{H} 4$ dimers from ASF1 to the HIRA complex is indeed spatially and temporally uncoupled from the proper nucleosome assembly process. Once loaded with histones, the HIRA complex must reach the sperm nucleus and target sperm DNA regions that become exposed following SNBP eviction. This likely involves the DNA binding property of the HIRA/YEM complex $[28,51]$ in a way similar to the nucleosome gap filling mechanism already mentioned. Assembly of paternal chromatin is, however, unique as it occurs at the scale of the whole male nucleus and takes place in the very large volume of the egg cell. The HIRA complex preloading with $\mathrm{H} 3.3-\mathrm{H} 4$ dimers could facilitate the rapid and efficient assembly of paternal chromatin, which must take place within minutes after the delivery of the sperm nucleus in the egg cytoplasm.

How the partitioning of $\mathrm{H} 3.2-\mathrm{H} 4$ and $\mathrm{H} 3.3-\mathrm{H} 4$ histone dimers on ASF1 is established before zygote formation remains, however, poorly understood. A recent study has proposed that the higher abundance of ASF1 in H3.3 complexes purified from early Drosophila embryos could indicate a higher affinity of ASF1 for H3.3 over H3.2 [52]. If true, this difference could fit well with the role of ASF1 in providing H3.3-H4 dimers to the HIRA complex at fertilization. In Drosophila eggs, the absence of ASF1 in the male pronucleus at least indicates that HIRA and ASF1 no longer interact during nucleosome assembly. Interestingly, it has been recently shown that the specificity of the human HIRA complex for H3.3-H4 over $\mathrm{H} 3.1-\mathrm{H} 4$ is conferred by UBN1, and not by HIRA itself [53]. The UBN1-H3.3 binding involved specific residues of the UBN1 HRD domain, which are conserved in the Drosophila orthologous protein YEM [53]. In Drosophila, ASF1 has been previously shown to interact with YEM in co-immunoprecipitation experiments [54]. We thus speculate that YEM, through its interaction with ASF1-H3.3-H4 complexes, controls the specific handover of H3.3-H4 over H3.2-H4 to the HIRA complex in the cytoplasm of Drosophila eggs. In this context, the HIRA B domain could play a secondary role, perhaps by reinforcing this interaction.

\section{Conclusions}

Our functional characterization of ASF1 has established the role of this histone chaperone in the maintenance of chromatin integrity during female meiosis and the amplification of embryonic nuclei. This work also revealed that ASF1 is critically required to assist the HIRA complex during the assembly of paternal chromatin. ASF1 
provides $\mathrm{H} 3.3-\mathrm{H} 4$ histone dimers to the HIRA complex but itself does not reside in the male pronucleus during de novo chromatin assembly. The spatio-temporal uncoupling of histone loading on the HIRA complex and histone deposition on paternal DNA illustrates the adaptability of histone chaperones and chromatin assembly factors to control such a highly constrained chromatin assembly process. Further work should aim at understanding how this essential cooperation is orchestrated during egg activation and fertilization.

\section{Methods}

\section{Drosophila stocks}

Flies were raised at $25{ }^{\circ} \mathrm{C}$ (unless otherwise indicated) on standard cornmeal-agar-yeast medium. The $w^{1118}$ strain was used as a wild-type control. The following stocks were obtained from the Bloomington Drosophila Stock Center (simplified genotypes are given): asf $1^{1}$ (\#9547) [30], asf $1^{2}$ (\#9548) [30], P\{TRiP.GL00349\} attP2 (yem shRNA; \#35425), P\{TRiP. GL00257\}attP2 (Hira shRNA; \#35346), P\{TRiP.HMC03937\}attP40 (asf1 shRNA; \#55250), P\{TRiP. GL00171\}attP2 (asf1 shRNA; \#35273), P\{GAL4-vg.M\}2 (\#6819; vg-Gal4), P\{Act5CGAL4\} $\}^{25 F O 1}$ (\#4414), P\{otu-GAL4::VP16.R\}1; P\{GAL4nos.NGT\}40; P\{GAL4::VP16-nos.UTR\}MVD1 (Maternal Triple Driver or "MTD-Gal4"; \#31777), P\{GAL4::VP16nos.UTR\}MVD1 ("nos-Gal4"; \#4937). Other stocks are: $w^{1118} \mathrm{Hira}^{\text {ssm }} /$ FM7c [55], $w^{1118} \mathrm{Hira}^{\text {HR1 }} /$ FM7 h [27], $P[w+, g$-EGFP-cid]III.2 [35], p1803/FM7i [40], P[w+, Hira::3xFlag]II/CyO [23] and P[w+, Hira $\Delta B:: 3 x$ Flag]I/ FM7 (this study).

\section{Transgenic constructs \\ $P[w+$, Hira $\Delta B:: 3 x F l a g]$}

The plasmid P[w+, Hira::3xFlag] [23] was used as a template to amplify a 2440-bp fragment from -400 upstream the ATG to +2040 by PCR using primers HIRABglII (5'CGCCCGCGGAAAGATCTATTCTTATATG-3') and HIRAPEPT13' (5'-TGGATCCGCGCAATGCACTGC AGAACT-3'). The PCR fragment was cloned into the PGEM-T vector (Promega) and verified by sequencing. The resulting plasmid was used as a template to generate a deletion in the region corresponding to the $\mathrm{B}$ domain minimal region [43]. Two mutagenic primers DeletBfwd (5'-AGCGACCCATTAGTAAACAAACGGAAACGC ACGAAGATGGACCCACATCGCTGA-3') and DeletBrev (5'-TCAGCGATGTGGGTCCATCTTCGTGCG TTTCCGTTTGTTTACTAATGGGTCGCT-3') were used to delete a $48 \mathrm{bp}$ region (corresponding to aa 428 to 443 of the HIRA protein) using the Quickchange II site directed mutagenesis kit (Stratagene). The construct was verified by sequencing, digested by BglII and reinserted into $P[w+$, Hira::3xFlag] to generate the $P[w+$,
Hira $\Delta B:: 3 x$ Flag]. Transgenic flies were obtained by standard P-mediated germline transformation.

\section{$P[w+, g-a s f 1:: V 5] a t t B$}

A 1920 -bp fragment covering de $5^{\prime}$ regulatory sequences and the complete coding sequence (CDS) of asf1 was amplified by PCR from $y w$ genomic DNA and subcloned into the pGEM-T vector (Promega). Primers used were: 5'CGGCGGCCGCCAGTCCACATCACA3', which introduces a NotI restriction site and 5' TTGGTACCT CACGTAGAATCGAGACCGAGGAGAGGGTTAGGG ATAGGCTTACCACATTCCATGGCCAGTGAGT $\quad 3^{\prime}$ which introduces a V5 tag, stop codon and KpnI restriction site. Similarly, a 836-bp fragment covering the downstream regulatory region of asf 1 was amplified by PCR from $y w$ genomic DNA and subcloned into the pGEM$T$ vector using the following primers: $5^{\prime}$ GGTACCGAG CTGCCGGCAGTCCGAAC3' and 5'TTCTAGACT GATCCGAGGGCAACTGCC $3^{\prime}$ containing $K p n \mathrm{I}$ and $\mathrm{Xba \textrm {I }}$ restriction sites, respectively. Both fragments were excised and cloned into the pattB vector (www.flyc3 1.org) using NotI, KpnI and KpnI, XbaI restriction sites, respectively.

\section{$P[w+$, UASP-asf1::V5]attB}

The asf1 full-length coding sequences was amplified by PCR from $y w$ genomic DNA using the following primers: 5'GAGGTACCATGGCCAAGGTGCACATCAC $3^{\prime}$ and 5'TTGGTACCTCACGTAGAATCGAGACCGAGG AGAGGGTTAGGGATAGGCTTACCACATTCCATG GCCAGTGAGT3'. Both primers include a KpnI restriction site subsequently used for cloning into the $p U A S P$ attB vector (www.flyc31.org).

All asf1::V5 transgenes were integrated in the PBac\{attP-3B\}VK00031 platform (62E1) using PhiC31mediated transformation [56].

\section{Fertility tests}

To obtain KD females, virgin shRNA transgenic females were mass crossed with transgenic Gal4 males at $25{ }^{\circ} \mathrm{C}$ and females of the desired genotype were recovered in the F1 progeny. To measure fertility, virgin females were aged for 3 days at $25{ }^{\circ} \mathrm{C}$ in the presence of males and were then allowed to lay eggs on standard medium for $24 \mathrm{~h}$. Embryos were counted and then let to develop at least $36 \mathrm{~h}$ at $25^{\circ} \mathrm{C}$. Embryo hatching rates were then calculated.

\section{Protein immunoprecipitation and Western blotting}

About $50 \mu \mathrm{l}$ of adult ovaries were dissected and homogenized in 2 volumes of lysis buffer [ $20 \mathrm{mM}$ Hepes $\mathrm{pH} 7.9$, $100 \mathrm{mM} \mathrm{KCl}, 0.1 \mathrm{mM}$ EDTA, $0.1 \mathrm{mM}$ EGTA, 5\% Glycerol, 0.05\% Igepal (Sigma Aldrich \#9002-93-1) with a 
protease inhibitor cocktail (Roche \#04693159001). Protein extracts were cleared by centrifugation and stored at $-80^{\circ} \mathrm{C}$.

Embryos were collected every $60 \mathrm{~min}$ and dechorionated in bleach. Protein extracts were prepared from approximately $20 \mu \mathrm{l}$ of embryos, as described for gonads.

For co-immunoprecipitation experiments, protein extracts were prepared from ovaries of females expressing ASF1 and/or HIRA proteins fused with C-terminal V5 or 3xFLAG tags, respectively. $100 \mu \mathrm{g}$ of protein extracts were incubated with $30 \mu \mathrm{l}$ of prewashed anti-V5 agarose beads (Sigma Aldrich \#A7345) or anti-FLAG M2 affinity gel (Sigma Aldrich \#A2220) overnight at $4{ }^{\circ} \mathrm{C}$. The beads were extensively washed with washing buffer [20 mM Hepes pH 7.9, $100 \mathrm{mM} \mathrm{KCl,} 200 \mathrm{mM} \mathrm{NaCl}$, 0.1 mM EDTA, 0.1 mM EGTA, 5\% Glycerol, 0.05\% Igepal CA-630].

Before loading, samples were boiled in Laemmli buffer containing DTT. Electrophoresis was carried out on $10 \%$ SDS polyacrylamide gel, and Western blotting was performed using the ECL prime Western blotting detection reagent and following the manufacturer's instructions (GE Healthcare). The antibodies were: mouse monoclonal anti- $\alpha$-Tubulin (Sigma Aldrich \#T9026, 1:500), rabbit polyclonal anti-ASF1 (1:2000), rabbit polyclonal anti-V5 (Invitrogen \#R960-25, 1:500), mouse monoclonal antiFlag M2 (Sigma Aldrich \#F3165, 1:25000), HRP-conjugated goat anti-mouse (Biorad \#170-5047; 1:50 000) and peroxidase-conjugated goat anti-rabbit (ThermoScientific \#32460; 1:20 000). The experiment was performed twice with similar results.

\section{Immunofluorescence and microscopy}

Embryos were collected on grape juice-agar plates, dechorionated in bleach, fixed in a 1:1 heptane/methanol mixture and stored at $-20{ }^{\circ} \mathrm{C}$. For immunostaining experiments, embryos were washed three times (10 $\mathrm{min}$ each) in PBS, $0.1 \%$ Triton X100. Embryos were then incubated with primary antibodies in the same buffer on a wheel overnight at $4{ }^{\circ} \mathrm{C}$ and washed three times $(20 \mathrm{~min}$ each) in PBS, $0.1 \%$ Triton X100. Incubations with secondary antibodies were performed identically. For propidium iodide staining, embryos were incubated for $1 \mathrm{~h}$ in a $2 \mathrm{mg}$ per $\mathrm{ml}$ RNAse A solution at $37^{\circ} \mathrm{C}$. Embryos were finally mounted in mounting medium (Dako) containing propidium iodide or DAPI.

Ovaries were dissected in PBS-Triton 0,1\% and fixed at room temperature in $4 \%$ formaldehyde in PBS for $25 \mathrm{~min}$. Immunofluorescence was performed as for embryos except for the secondary antibodies that were incubated four hours at room temperature. Ovaries were then stained with propidium iodide and mounted as described above.
Primary antibodies used were: mouse monoclonal anti-GFP (Roche \#118144600001; 1:200), mouse monoclonal anti-V5 (Invitrogen \#R960-25, 1:500), rabbit polyclonal anti-polyacetylated Histone $\mathrm{H} 4$ (Millipore \#06-589; 1:250), mouse monoclonal anti-histones (Millipore \#MABE71; 1:1000), rabbit polyclonal anti-Hira (PG1 purified, 1:500), rabbit polyclonal anti-ASF1 [30] (A gift from F. Karch; 1:1000) and mouse monoclonal anti-PCNA (Abcam \#ab29, 1:1000). Secondary antibodies were used at a 1:1000 dilution and included goat anti-mouse and goat anti-rabbit antibodies conjugated to AlexaFluor (Jackson ImmunoResearch). Images were acquired on a LSM 510 META (Carl Zeiss), and the images were processed with Adobe Photoshop and Adobe Illustrator. At least two independent experiments were performed for each immunostaining.

\section{Additional files}

Additional file 1: Fig. S1. asf1 KD embryos develop without detectable levels of ASF1. Confocal images of a nuclear cycle 10 control embryo (left) and a nuclear cycle 11 asf1 KD embryo (right) stained for DNA (red) and ASF1 (green). ASF1 is not detected in the nuclei of asf1 KD embryo. Scale bar: $20 \mu \mathrm{m}$.

Additional file 2: Fig. S2. Nuclear defects in early asf1 KD embryos. Confocal images of control and asf1 KD embryos at the indicated stage stained with anti-histone antibodies. Yellow arrows indicate chromatin bridges. Red arrow indicates disintegrated nuclei. Embryos are delineated by a dashed line. PB: Polar bodies. Scale bar: $50 \mu \mathrm{m}$.

Additional file 3: Table S1. Developmental arrest of embryos.

Additional file 4: Fig. S3. asf1 KD embryos lack paternal chromosomes. Late control and asf1 KD embryos fathered by GFP.:.cid transgenic males. Zygotic expression of the paternal centromeric marker is only detected in nuclei of control embryos (arrows in left inset). $n=140$ for control embryos and $n=40$ for asf1 KD embryos. Scale bar: $50 \mu \mathrm{m}$.

Additional file 5: Fig. S4. ASF1::V5 is not detected in the decondensing male pronucleus. a: An egg in metaphase of meiosis II from a g-asfi::V5 transgenic female stained with anti-V5 antibodies. Scale bar: $50 \mu \mathrm{m}$. b: Pronuclear migration. Scale bar: $10 \mu \mathrm{m}$. c: ASF 1::V5 is incorporated in both pronuclei at the onset of DNA replication. Scale bar: $10 \mu \mathrm{m}$. d: Pronuclei stained with anti-V5 antibodies during apposition. Scale bar: $10 \mu \mathrm{m}$.

\section{Abbreviations}

ASF1: Anti-Silencing Factor-1; RC: Replication-Coupled; RI: ReplicationIndependent; CAF-1: Chromatin Assembly Factor-1; shRNA: small hairpin RNA; KD: knocked-down; HIRA: Histone Regulator-A; ATRX: alpha thalassemia/ mental retardation syndrome X-linked; XNP: X-linked nuclear protein; UBN1: ubinuclein-1; SNBP: sperm nuclear basic protein; YEM: yemanuclein; UAS: upstream activating sequence; UTR: untranslated region; GV: germinal vesicle; CID: centromere identifier; MCM2: minichromosome maintenance-2; HRP: horseradish peroxidase; GFP: green fluorescent protein; PCNA: proliferating cell nuclear antigen.

\section{Authors' contributions}

$\mathrm{BH}$ and $\mathrm{BL}$ designed the experiments. $\mathrm{BH}$ and $\mathrm{LS}$ performed most of the experiments. EB generated the $P[w+$, Hira $\triangle B:: 3 x$ Flag $]$ transgene and performed the corresponding rescue experiments. $\mathrm{BH}$ and $\mathrm{BL}$ analyzed the data. $\mathrm{BL}$ wrote the manuscript and $\mathrm{BH}$ revised it. All authors read and approved the final manuscript. 


\section{Acknowledgements}

We thank Nathalie Dostatni, Stefan Heidmann and François Karch for fly stocks and antibodies. We also thank Raphaëlle Dubruille for her helpful comments on the manuscript.

\section{Competing interests}

The authors declare that they have no competing interests.

\section{Availability of data and materials}

Materials generated for this study (Drosophila stocks and plasmids) are available upon request. No datasets were generated or analyzed during the current study.

\section{Consent for publication}

Not Applicable.

\section{Ethics approval and consent to participate}

Not Applicable.

\section{Funding}

This work was supported by a grant from the Agence Nationale de la Recherche (ZygoPat-ANR-12-BSV6-0014).

\section{Publisher's Note}

Springer Nature remains neutral with regard to jurisdictional claims in published maps and institutional affiliations.

Received: 27 February 2018 Accepted: 24 April 2018

Published online: 11 May 2018

\section{References}

1. Polo SE, Almouzni G. Chromatin assembly: a basic recipe with various flavours. Curr Opin Genet Dev. 2006;16:104-11.

2. Luger K, Dechassa ML, Tremethick DJ. New insights into nucleosome and chromatin structure: an ordered state or a disordered affair? Nat Rev Mol Cell Biol. 2012;13:436-47.

3. Hammond CM, Strømme CB, Huang H, Patel DJ, Groth A. Histone chaperone networks shaping chromatin function. Nat Rev Mol Cell Biol. 2017;18:1-19.

4. Burgess RJ, Zhang Z. Histone chaperones in nucleosome assembly and human disease. Nat Struct Mol Biol. 2013;20:14-22.

5. Gurard-Levin ZA, Quivy J-P, Almouzni G. Histone chaperones: assisting histone traffic and nucleosome dynamics. Annu Rev Biochem. 2014:83:487-517.

6. English CM, Maluf NK, Tripet B, Churchill MEA, Tyler JK. ASF1 binds to a heterodimer of histones $\mathrm{H} 3$ and $\mathrm{H} 4$ : a two-step mechanism for the assembly of the $\mathrm{H} 3-\mathrm{H} 4$ heterotetramer on DNA. Biochemistry. 2005:44:13673-82.

7. Mousson F, Lautrette A, Thuret J-Y, Agez M, Courbeyrette R, Amigues B, et al. Structural basis for the interaction of Asf1 with histone $\mathrm{H} 3$ and its functional implications. Proc Natl Acad Sci USA. 2005;102:5975-80.

8. Schulz LL, Tyler JK. The histone chaperone ASF1 localizes to active DNA replication forks to mediate efficient DNA replication. FASEB J. 2006;20:488-90.

9. English CM, Adkins MW, Carson JJ, Churchill MEA, Tyler JK. Structural basis for the histone chaperone activity of Asf1. Cell. 2006;127:495-508.

10. Tyler JK, Collins KA, Prasad-Sinha J, Amiott E, Bulger M, Harte PJ, et al. Interaction between the drosophila CAF-1 and ASF1 chromatin assembly factors. Mol Cell Biol. 2001;21:6574-84

11. Tagami H, Ray-Gallet D, Almouzni G, Nakatani Y. Histone H3.1 and H3.3 complexes mediate nucleosome assembly pathways dependent or independent of DNA synthesis. Cell. 2004;116:51-61.

12. Green EM, Antczak AJ, Bailey AO, Franco AA, Wu KJ, Yates JR, et al. Replication-independent histone deposition by the HIR complex and Asf1. Curr Biol. 2005;15:2044-9.

13. Zhang R, Poustovoitov MV, Ye X, Santos HA, Chen W, Daganzo SM, et al. Formation of MacroH2A-containing senescence-associated heterochromatin foci and senescence driven by ASF1a and HIRA. Dev Cell. 2005;8:19-30.

14. Ransom M, Dennehey BK, Tyler JK. Chaperoning histones during DNA replication and repair. Cell. 2010;140:183-95.

15. Natsume R, Eitoku M, Akai Y, Sano N, Horikoshi M, Senda T. Structure and function of the histone chaperone CIA/ASF1 complexed with histones H3 and H4. Nature. 2007;446:338-41.

16. Wang $H$, Wang $M$, Yang $N$, Xu R-M. Structure of the quaternary complex of histone $\mathrm{H} 3-\mathrm{H} 4$ heterodimer with chaperone ASF1 and the replicative helicase subunit MCM2. Protein Cell. 2015;6:693-7.

17. Groth A, Corpet A, Cook AJL, Roche D, Bartek J, Lukas J, et al. Regulation of replication fork progression through histone supply and demand. Science. 2007;318:1928-31.

18. Richet N, Liu D, Legrand P, Velours C, Corpet A, Gaubert A, et al. Structural insight into how the human helicase subunit MCM2 may act as a histone chaperone together with ASF1 at the replication fork. Nucl Acids Res. 2015:43:1905-17

19. Yang J-H, Choi J-H, Jang H, Park J-Y, Han J-W, Youn H-D, et al. Histone chaperones cooperate to mediate Mef2-targeted transcriptional regulation during skeletal myogenesis. Biochem Biophys Res Commun. 2011;407:541-7.

20. Schneiderman Jl, Orsi GA, Hughes KT, Loppin B, Ahmad K. Nucleosomedepleted chromatin gaps recruit assembly factors for the $\mathrm{H} 3.3$ histone variant. Proc Natl Acad Sci USA. 2012;109:19721-6.

21. Pchelintsev NA, McBryan T, Rai TS, van Tuyn J, Ray-Gallet D, Almouzni G, et al. Placing the HIRA histone chaperone complex in the chromatin landscape. Cell Rep. 2013;3:1012-9.

22. Yamane K, Mizuguchi T, Cui B, Zofall M, Noma K-I, Grewal SIS. Asf1/HIRA facilitate global histone deacetylation and associate with HP1 to promote nucleosome occupancy at heterochromatic loci. Mol Cell. 2011;41:56-66.

23. Loppin B, Bonnefoy E, Anselme C, Laurençon A, Karr TL, Couble P. The histone H3.3 chaperone HIRA is essential for chromatin assembly in the male pronucleus. Nature. 2005;437:1386-90.

24. Inoue A, Zhang Y. Nucleosome assembly is required for nuclear pore complex assembly in mouse zygotes. Nat Struct Mol Biol. 2014;21:609-16.

25. Lin C-J, Koh FM, Wong P, Conti M, Ramalho-Santos M. Hira-mediated $\mathrm{H} 3.3$ incorporation is required for DNA replication and ribosomal RNA transcription in the mouse zygote. Dev Cell. 2014;30:268-79.

26. Tirmarche S, Kimura S, Dubruille R, Horard B, Loppin B. Unlocking sperm chromatin at fertilization requires a dedicated egg thioredoxin in Drosophila. Nat Commun. 2016;7:13539.

27. Bonnefoy E, Orsi GA, Couble P, Loppin B. The essential role of drosophila HIRA for de novo assembly of paternal chromatin at fertilization. PLoS Genet. 2007;3:e182.

28. Orsi GA, Algazeery A, Meyer RE, Capri M, Sapey-Triomphe LM, Horard B, et al. Drosophila yemanuclein and HIRA cooperate for de novo assembly of H3.3-containing nucleosomes in the male pronucleus. PLoS Genet. 2013;9:e1003285.

29. Horard B, Loppin B. Histone storage and deposition in the early Drosophila embryo. Chromosoma. 2015;124:163-75.

30. Moshkin YM, Armstrong JA, Maeda RK, Tamkun JW, Verrijzer P, Kennison JA, et al. Histone chaperone ASF1 cooperates with the Brahma chromatin-remodelling machinery. Genes Dev. 2002;16:2621-6.

31. Ni J-Q, Zhou R, Czech B, Liu L-P, Holderbaum L, Yang-Zhou D, et al. A genome-scale shRNA resource for transgenic RNAi in Drosophila. Nat Methods. 2011;8:405-7.

32. Yamaguchi M, Date T, Matsukage A. Distribution of PCNA in Drosophila embryo during nuclear division cycles. J Cell Sci. 1991;100:729-33.

33. Yamaguchi M, Hirose F, Nishimoto $Y$, Naruge T, Ikeda M, Hachiya T, et al. Expression patterns of DNA replication enzymes and the regulatory factor DREF during Drosophila development analyzed with specific antibodies. Biol Cell. 1995;85:147-55.

34. Edgar BA, Kiehle CP, Schubiger G. Cell cycle control by the nucleo-cytoplasmic ratio in early Drosophila development. Cell. 1986;44:365-72.

35. Schuh M, Lehner CF, Heidmann S. Incorporation of Drosophila CID/CENP$A$ and CENP-C into centromeres during early embryonic anaphase. Curr Biol. 2007; 17:237-43.

36. Rathke C, Baarends WM, Awe S, Renkawitz-Pohl R. Chromatin dynamics during spermiogenesis. Biochim Biophys Acta. 2014;1839:155-68.

37. Loppin B, Dubruille R, Horard B. The intimate genetics of Drosophila fertilization. Open Biol. 2015;5:150076. 
38. Loppin B, Berger F, Couble P. The Drosophila maternal gene sésame is required for sperm chromatin remodeling at fertilization. Chromosoma. 2001;110:430-40

39. Orsi GA, Couble P, Loppin B. Epigenetic and replacement roles of histone variant H3.3 in reproduction and development. Int J Dev Biol. 2009;53:231-43.

40. Klapholz B, Dietrich BH, Schaffner C, Hérédia F, Quivy J-P, Almouzni G, et al. CAF-1 is required for efficient replication of euchromatic DNA in Drosophila larval endocycling cells. Chromosoma. 2009:118:235-48.

41. Ray-Gallet D, Woolfe A, Vassias I, Pellentz C, Lacoste N, Puri A, et al. Dynamics of histone $\mathrm{H} 3$ deposition in vivo reveal a nucleosome gapfilling mechanism for H3.3 to maintain chromatin integrity. Mol Cell. 2011:44:928-41.

42. Kirov N, Shtilbans A, Rushlow C. Isolation and characterization of a new gene encoding a member of the HIRA family of proteins from Drosophila melanogaster. Gene. 1998;212:323-32.

43. Tang Y, Poustovoitov MV, Zhao K, Garfinkel M, Canutescu A, Dunbrack $R$, et al. Structure of a human ASF1a-HIRA complex and insights into specificity of histone chaperone complex assembly. Nat Struct Mol Biol. 2006;13:921-9.

44. Mejlvang J, Feng Y, Alabert C, Neelsen KJ, Jasencakova Z, Zhao X, et al. New histone supply regulates replication fork speed and PCNA unloading. J Cell Biol. 2014;204:29-43.

45. Tyler JK, Adams CR, Chen SR, Kobayashi R, Kamakaka RT, Kadonaga JT. The RCAF complex mediates chromatin assembly during DNA replication and repair. Nature. 1999;402:555-60.

46. Delabaere L, Orsi GA, Sapey-Triomphe L, Horard B, Couble P, Loppin B. The spartan ortholog maternal haploid is required for paternal chromosome integrity in the Drosophila zygote. Curr Biol. 2014;24:2281-7.

47. Ray-Gallet D, Quivy J-P, Silljé HWW, Nigg EA, Almouzni G. The histone chaperone Asf1 is dispensable for direct de novo histone deposition in Xenopus egg extracts. Chromosoma. 2007;116:487-96.
48. Jasencakova Z, Groth A. Restoring chromatin after replication: how new and old histone marks come together. Semin Cell Dev Biol. 2010;21:231-7.

49. Shintomi K, Inoue F, Watanabe H, Ohsumi K, Ohsugi M, Hirano T. Mitotic chromosome assembly despite nucleosome depletion in Xenopus egg extracts. Science. 2017;356:1284-7.

50. Adam S, Polo SE, Almouzni G. Transcription recovery after DNA damage requires chromatin priming by the $\mathrm{H} 3.3$ histone chaperone HIRA. Cell. 2013;155:94-106.

51. Aitt-Ahmed O, Bellon B, Capri M, Joblet C, Thomas-Delaage M. The yemanuclein-alpha: a new Drosophila DNA binding protein specific for the oocyte nucleus. Mech Dev. 1992;37:69-80.

52. Fromental-Ramain C, Ramain P, Hamiche A. The Drosophila DAXX-Like protein (DLP) cooperates with ASF1 for H3.3 deposition and heterochromatin formation. Mol Cell Biol. 2017;37:e00597-16.

53. Ricketts DM, Frederick B, Hoff H, Tang Y, Schultz DC, Singh Rai T, et al. Ubinuclein-1 confers histone H3.3-specific-binding by the HIRA histone chaperone complex. Nat Commun. 2015;6:7711.

54. Moshkin YM, Kan TW, Goodfellow H, Bezstarosti K, Maeda RK, Pilyugin M, et al. Histone chaperones ASF1 and NAP1 differentially modulate removal of active histone marks by LID-RPD3 complexes during NOTCH silencing. Mol Cell. 2009;35:782-93.

55. Loppin B, Docquier M, Bonneton F, Couble P. The maternal effect mutation sésame affects the formation of the male pronucleus in Drosophila melanogaster. Dev Biol. 2000;222:392-404.

56. Bischof J, Maeda RK, Hediger M, Karch F, Basler K. An optimized transgenesis system for Drosophila using germ-line-specific phiC31 integrases. Proc Natl Acad Sci USA. 2007;104:3312-7.
Ready to submit your research? Choose BMC and benefit from:

- fast, convenient online submission

- thorough peer review by experienced researchers in your field

- rapid publication on acceptance

- support for research data, including large and complex data types

- gold Open Access which fosters wider collaboration and increased citations

- maximum visibility for your research: over 100M website views per year

At BMC, research is always in progress.

Learn more biomedcentral.com/submissions 